\title{
Boundary layer emission and Z-track in the color-color diagram of luminous LMXBs
}

\author{
M. G. Revnivtsev ${ }^{1,2}$ and M. R. Gilfanov ${ }^{1,2}$ \\ 1 Max-Planck-Institute für Astrophysik, Karl-Schwarzschild-Str. 1, 85740 Garching bei München, Germany \\ e-mail: mikej@mpa-garching.mpg.de \\ 2 Space Research Institute, Russian Academy of Sciences, Profsoyuznaya 84/32, 117997 Moscow, Russia
}

Received 2 August 2005 / Accepted 20 February 2006

\begin{abstract}
Aims. We explore the accretion disk and boundary layer emission in bright neutron star LMXBs and their dependence on the mass accretion rate.

Methods. We used Fourier-frequency resolved spectroscopy of the archival RXTE data.

Results. We demonstrate that Fourier-frequency resolved spectra of atoll and Z-sources are identical, despite significant differences in their average spectra and luminosity (by a factor of $\sim 10-20$ ). This result fits in the picture we suggested earlier, namely that the $f \gtrsim 1 \mathrm{~Hz}$ variability in luminous LMXBs is primarily due to variations in the boundary layer luminosity. In this picture the frequency-resolved spectrum equals the boundary layer spectrum, which therefore can be straightforwardly determined from the data. This boundary layer spectrum is approximated well by the saturated Comptonization model, and its high energy cut-off follows a $k T \approx 2.4 \mathrm{keV}$ blackbody. Its independence from the global mass-accretion rate lends support to a theoretical suggestion, that the boundary layer is supported by radiation pressure. With this assumption we constrain the gravity on the neutron star surface, its mass and radius. Equipped with the knowledge of the boundary layer spectrum, we attempt to relate the motion along the Z-track to changes of physically meaningful parameters. Our results suggest that the contribution of the boundary layer to the observed emission decreases along the Z-track from conventional $\sim 50 \%$ on the horizontal branch to a rather small number on the normal branch. This decrease can be caused, for example, by obscuration of the boundary layer by the geometrically thick accretion disk at $\dot{M} \sim \dot{M}_{\text {Edd }}$. Alternatively, this can indicate a significant change in the structure of the accretion flow at $\dot{M} \sim \dot{M}_{\text {Edd }}$ and the disappearance of the boundary layer as a distinct region of the significant energy release associated with the neutron star surface.
\end{abstract}

Key words. accretion, accretion disks - stars: binaries: general - X-rays: general - X-rays: binaries stars: novae, cataclysmic variables

\section{Introduction}

Accreting neutron stars in low mass X-ray binaries (LMXB) are among the most luminous compact $\mathrm{X}$-ray sources in the Galaxy. At least several of them have luminosities exceeding $\sim$ few $\times 10^{38} \mathrm{erg} / \mathrm{s}$, and they presumably accrete matter at a level close to the critical Eddington accretion rate. Early observations of these sources (e.g. Toor et al. 1970) revealed rather soft X-ray spectra, indicating that their X-ray emission is predominantly formed in the optically thick media. Similar to accreting black holes, at lower X-ray luminosities (lower mass accretion rates), $L_{\mathrm{x}} \lesssim 5 \times 10^{36} \mathrm{erg} / \mathrm{s}$, neutron stars undergo a transition to the hard spectral state (e.g. Barret 2001). The energy spectra in this state point toward the low optical depth in the emission region.

In the soft spectral state, the commonly accepted picture of accretion at values of the accretion rate that are not too extreme has two main ingredients - the accretion disk (AD) and the boundary layer (BL). While matter in the disk rotates with nearly Keplerian velocities, in the boundary layer it decelerates down to the spin frequency of the neutron star and settles onto its surface. For the typical neutron star spin frequency $(\$ 500-700 \mathrm{~Hz})$, comparable amounts of energy are released in these two regions (Sunyaev \& Shakura 1986; Sibgatullin \& Sunyaev 2000). This picture is based on rather obvious qualitative expectations, as well as on more sophisticated theoretical considerations and numerical modeling (Sunyaev \& Shakura 1986; Kluzniak 1988; Inogamov \& Sunyaev 1999; Sibgatullin \& Sunyaev 2000). This has been receiving, however, little direct observational confirmation. Due to the similarity of the spectra of the accretion disk and boundary layer, the total spectrum has a smooth curved shape, which is difficult to decompose into separate spectral components (Mitsuda et al. 1984; White et al. 1988; Di Salvo et al. 2001; Done et al. 2002). This made it difficult to apply physically motivated spectral models to the description of observed spectra of luminous neutron stars, in spite of a very significant increase in the sensitivity of X-ray instruments. A possible solution was suggested by early results by Mitsuda et al. (1984), which demonstrated the potential of using the combined spectral and variability information.

Recently, Gilfanov et al. (2003) analyzed spectral variability in luminous LMXBs and showed that in these sources aperiodic and quasi periodic variability on $\sim \mathrm{s}-\mathrm{ms}$ time scales is primarily caused by variations in the luminosity of the boundary layer. Its spectral shape remains nearly constant in the course of the luminosity variations and is represented by the Fourierfrequency resolved spectrum. Moreover, in the considered range $\dot{M} \sim(0.1-1) \dot{M}_{\text {Edd }}\left(\dot{M}_{\text {Edd }}\right.$ is the critical Eddington mass accretion rate), it depends weakly on the global mass accretion rate and in the limit $\dot{M} \sim \dot{M}_{\text {Edd }}$ is close to the Wien spectrum with 
$k T \sim 2.4 \mathrm{keV}$. Such behavior accords with the predictions of the model by Inogamov \& Sunyaev (1999), namely, that at sufficiently high accretion rates, $\dot{M}>0.1 \dot{M}_{\text {Edd }}$, the boundary layer is radiation-pressure dominated, and the local radiation flux is close to the critical Eddington value. Increase of the mass accretion rate leads to the increase of the emitting area of the BL, while its vertical structure changes little (Inogamov \& Sunyaev 1999).

In this paper we further explore the behavior of the boundary layer and the accretion disk emission in a number of bright neutron star LMXBs (both Z and atoll sources) and qualitatively describe their evolution with change in the mass accretion rate. Our study is based on the results of Gilfanov et al. (2003), namely, that the shape of the boundary layer spectrum is adequately represented by the frequency-resolved spectrum (energy spectrum of the variable part of the emission) at the Fourier frequencies above $f \gtrsim 1 \mathrm{~Hz}$. We concentrate on neutron star LMXBs in the soft/high spectral state with luminosities $L_{X} \gtrsim 0.5-1 \times 10^{37} \mathrm{erg} / \mathrm{s}$. Sources in this state have a very important property - their frequency-resolved energy spectra do not depend on the Fourier frequency. It is this property that allows us to decompose the averaged energy spectrum of the sources into two components. In a hard spectral state, the variability is significantly more complicated (see e.g. Revnivtsev et al. 1999, 2000), so we leave it for later studies.

\section{Data}

For our study we used data of the RXTE observatory (Bradt et al. 1993), which combines large collecting area with high time resolution. We studied all Z-sources except for GX 349+2, which was on the "flaring" branch of its color-color diagram during the majority of our RXTE observations, supposedly corresponding to super-Eddington mass accretion rates (Hasinger \& van der Klis 1989). The behavior of sources at such high accretion rates is more complex and is beyond the scope of this paper. The remaining five $\mathrm{Z}$ sources are: Cyg X-2, GX 340+0, Sco X-1, GX 5-1, and GX 17+2. For these sources we excluded the time intervals corresponding to the "flaring" branch of their color-color diagram. The atoll sources were represented in our sample by 4U1608-52 and 4U1820-30 in their soft/high spectral state, showing high rapid flux variability.

For the data reduction we used standard programs of package HEASOFT 5.3 in accordance with the RXTE Guest Observer Facility recommendations. We corrected the obtained energy spectra for the deadtime and the pileup effects (http:// lheawww.gsfc.nasa.gov/docs/xray/xte/pca/). All subsequent spectral approximations included interstellar absorption, which was fixed at values: $N_{\mathrm{H}}=6 \times 10^{22} \mathrm{~cm}^{-2}$ for GX $340+0$ (Iaria et al. 2004), $0.2 \times 10^{22} \mathrm{~cm}^{-2}$ for Cyg X-2 (Kuulkers et al. 1997), $0.2 \times 10^{22} \mathrm{~cm}^{-2}$ for Sco X-1 (Kahn et al. 1984), $1.0 \times$ $10^{22} \mathrm{~cm}^{-2}$ for GX 17+2 (Langmeier et al. 1990), $3.0 \times 10^{22} \mathrm{~cm}^{-2}$ for GX 5-1 (Asai et al. 1994), $1.0 \times 10^{22} \mathrm{~cm}^{-2}$ for 4U1608-52 (Penninx et al. 1989), and $0.2 \times 10^{22} \mathrm{~cm}^{-2}$ for $4 \mathrm{U} 1820-30$ (Haberl et al. 1987). The luminosities of all $\mathrm{Z}$ sources are approximately $2 \times 10^{38} \mathrm{erg} \mathrm{s}^{-1}$, and for 4U1608-52 and 4U1820-30 approximately $10^{37} \mathrm{erg} \mathrm{s}^{-1}$.

The color-color diagrams (CCD) of these sources are presented in Fig. 1. For construction of the CCDs, we used energy channels 2.0-4.0 keV, 4.0-6.0 keV, 6.0-10.0 keV, and $10.0-15.0 \mathrm{keV}$. The hard and soft colors are ratios of the energy fluxes of sources in the abovementioned energy bands. Energy fluxes were computed from spectra averaged over $128 \mathrm{~s}$ intervals. All fluxes were corrected for absorption.

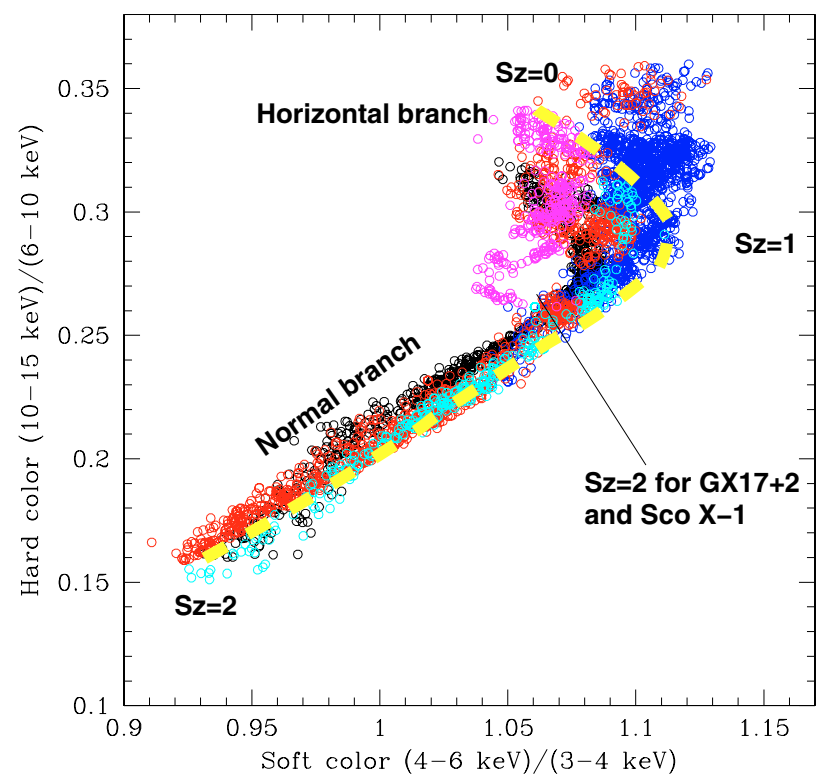

Fig. 1. Color-color diagram of all Z-sources except for GX 349+2. Only horizontal and normal branch data are shown. Colors were calculated as ratios of absorption corrected energy fluxes in the following energy channels $3.0-4.0 \mathrm{keV}, 4.0-6.0 \mathrm{keV}, 6.0-10.0 \mathrm{keV}, 10-15.0 \mathrm{keV}$. The dashed line shows the approximation to the Z-track by an arbitrary smooth curve. Turning points of the Z-track are marked with $S_{\mathrm{z}}=0,1,2$. Here and later, black points denote GX 340+0, red Cyg X-2, cyan - GX 5-1, magenta - Sco X-1, blue - GX 17+2.

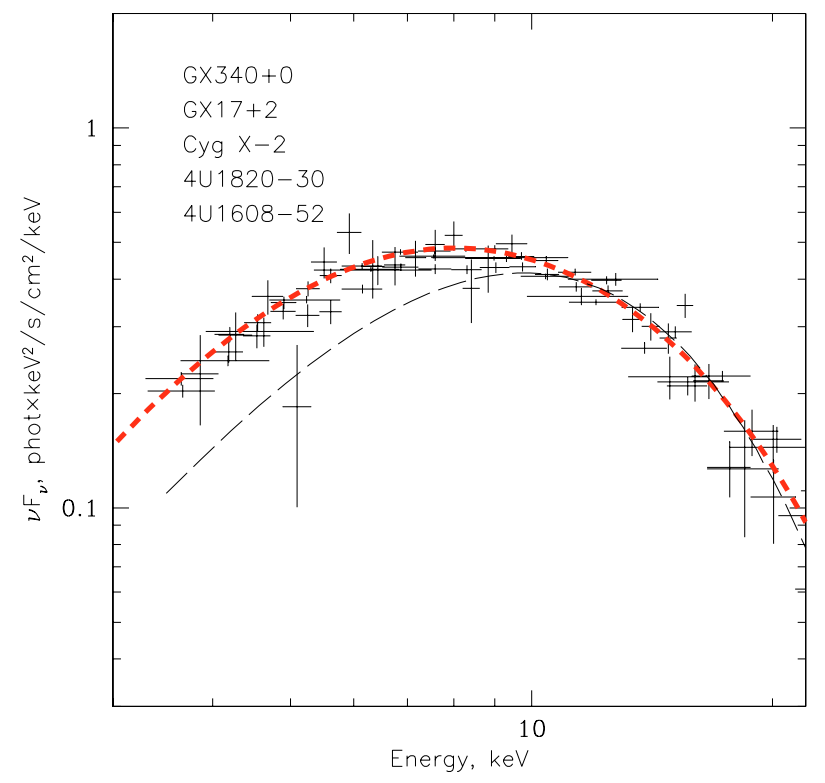

Fig. 2. Fourier-frequency resolved spectra ( $\approx$ boundary layer spectra) of sources from our sample. All spectra were corrected for the interstellar absorption. The thick dotted line shows the best fit Comptonization model with $k T_{\mathrm{s}}=1.5, k T_{\mathrm{e}}=3.3 \mathrm{keV}, \tau=5$. The thin dashed line shows the blackbody spectrum with temperature $k T_{\mathrm{bb}}=2.4 \mathrm{keV}$.

\section{Spectrum of the boundary layer}

The frequency resolved spectra (the energy spectrum of the variable part of the emission, Revnivtsev et al. 1999) computed at the frequencies $f \gtrsim 1-5 \mathrm{~Hz}$ are shown for five sources from our sample in Fig. 2. For $\mathrm{Z}$ sources we used only data on the horizontal branch of the CCD where the amplitude of variability at these frequencies is maximal. For Sco X-1 and GX 5-1 the 
RXTE achive does not contain high time resolution data for the horizontal branch with a sufficient number of energy channels. The frequency resolved spectrum of 4U1608-52 was taken from Gilfanov et al. (2003). For plotting purposes, the normalizations of all spectra were adjusted to match that of GX $340+0$.

The similarity of the spectra shown in Fig. 2 is remarkable, considering the significant difference in the average spectra and the factor of $\sim 10-20$ spread in the luminosity between atoll and Z-sources ( $\sim 0.1 \dot{M}_{\text {Edd }}$ and $\sim \dot{M}_{\text {Edd }}$, correspondingly). This behavior fits in the picture proposed by Gilfanov et al. (2003). As summarized in the Introduction, they showed, in particular, that the frequency-resolved spectra at these frequencies equal the spectrum of the boundary layer emission.

The shape of the frequency-resolved ( $\approx$ boundary layer) spectrum can be adequately described by the saturated Comptonization. For the sake of comparison with other results and for convenient parametrization of the BL spectrum, we used the Comptonization model of Titarchuk (1994). The best fit parameters of the model fitted simultaneousely in the $3-20 \mathrm{keV}$ range to all five spectra shown in the Fig. 2 are: temperature of seed photons $k T_{\mathrm{s}}=1.5 \pm 0.1$, temperature of electrons $k T_{\mathrm{e}}=3.3 \pm 0.4$ and the optical depth $\tau=5 \pm 1$ for slab geometry. The temperature of the blackbody spectrum describing the shape of the cutoff in the observed spectrum at energies $>13 \mathrm{keV}$ is $k T_{\mathrm{bb}}=2.4 \pm 0.1 \mathrm{keV}$.

It would be interesting to follow up on the results of Gilfanov et al. (2003) and to consider the evolution of the boundary spectrum along the $\mathrm{Z}$ track from the horizontal to normal branch. Unfortunately, apart from GX 340+0, that was already considered in their paper, the variability level on the normal branch of color-color diagram for the other four Z-sources is insufficient for obtaining frequency-resolved spectra with a reasonable signal-to-noise ratio.

\section{Constraints on NS mass and radius from the BL spectrum}

This independence of the boundary layer spectrum from the luminosity lends support to the theoretical predictions by Inogamov \& Sunyaev (1999) that the boundary layer is radiation-pressure supported. It means that, in the upper layers of the BL, only the radiation pressure counteracts the gravitational force. Therefore we can say that every unit area of the BL should emit Eddington surface flux. At lower massaccretion rates $\left(\dot{M} \sim 0.1 \dot{M}_{\text {Edd }}\right)$, the BL occupies only a part of the NS surface, giving rise to moderate total X-ray luminosities $\left(L_{\mathrm{x}} \sim 10^{37} \mathrm{erg} \mathrm{s}^{-1}\right)$. If the mass-accretion rate increases, the total BL luminosity approaches the Eddington limit for the NS $L_{\mathrm{Edd}} \sim 2 \times 10^{38} \mathrm{erg} \mathrm{s}^{-1}\left(1.4 M_{\odot}\right)^{-1}$. In this picture, the parameters of the BL emission can be used to determine the value of the Eddington flux limit on the surface of the neutron star. As the Eddington flux limit is uniquely determined by the neutron star surface gravity and by the atmospheric chemical composition, the neutron star mass and radius can be constrained.

If the boundary layer emitts true blackbody emission, the radiation flux of the unit area was determined only by its temperature. Therefore the observed shape of the BL spectrum, in particular the bestfit blackbody temperature, could be used to determine the value of the Eddington flux limit on the neutron star surface. This approach has been utilized in the context of the Eddington limited X-ray bursts (e.g. Goldman 1979;
Marshall 1982; Lewin et al. 1993; Titarchuk \& Shaposhnikov 2002). For fully ionized hydrogen atmosphere

$$
\frac{\sigma T^{4}}{c} \frac{\sigma_{\mathrm{T}}}{m_{\mathrm{p}}}=\frac{G M\left(1-R_{\mathrm{Sch}} / R\right)^{3 / 2}}{R^{2}}
$$

where $\sigma$ is the Stefan-Boltzmann constant, $\sigma_{\mathrm{T}}$ is the Thomson cross-section, $T$ is the blackbody temperature at infinity, $m_{\mathrm{p}}$ is the proton mass, $M$ is the mass and $R$ is the radius of the neutron star, $R_{\mathrm{Sch}}=2 G M / c^{2}$ is the Schwarzschild radius of the neutron star. In addition, one would have to take into account that the value of the Eddington flux is somewhat (by $\sim 10-20 \%$ ) reduced because of the action of the centrifugal force caused by the rotation of the boundary layer (Inogamov \& Sunyaev 1999). Rotation of the neutron star at this point is not very important unless it is very high (rotational frequency $\gtrsim 800-1000 \mathrm{~Hz}$ ).

In reality, scatterings are important in the atmosphere of the neutron star, therefore, the boundary layer spectrum will differ from the black body (e.g. London et al. 1986; Lewin et al. 1993). The radiation transfer problem in the atmosphere of the neutron star has been intensively investigated, in particular in the context of X-ray bursts. Numerical calculations show that the effects of scatterings can be approximately accounted for by introducing the spectral hardening factor that relates the color and the effective temperatures of the emission (Syunyaev \& Shakura 1974; London et al. 1986; Titarchuk 1994; Shimura \& Takahara 1995; Ross \& Fabian 1996). Typical values of the hardening factor are about $\sim 1.7$. We used this result in order to make simple estimates of the gravity on the neutron star surface and to constrain its mass and radius. In these calculations we assumed the color temperature of the boundary layer emission $T=2.4 \mathrm{keV}$ and considered the range of the hardening factor values of 1.6-1.8. We assumed that the centrifugal force reduces the Eddington flux limit in comparison with the non-rotating boundary layer by $20 \%$. We also took the finite height of the boundary/spreading layer into account, which is about $1 \mathrm{~km}$ (Inogamov \& Sunyaev 1999). The calculations were performed for Schwarzschild geometry, hydrogen atmosphere, and the result is shown in Fig. 3. The width of the shaded region is defined by the assumed range of the values of the hardening factor. Detailed modeling of the emergent spectrum of the BL will allow us to strongly diminish the size of uncertainty region.

Finally we note that the similarity of the spectral shape of the BL spectrum in different sources (Fig. 2) indicates that they have close values for the mass and radius, and in particular, that there is no significant difference in the surface gravity between atoll and Z-sources. It also shows that there are no significant differences caused by variations in the atmospheric chemical abundances between sources. We did not find a statistically significant difference between the ultracompact compact binary 4U1820-30 and other sources.

\section{Evolution along the Z-track}

In order to study the behavior of Z-sources in the color-color diagram, we considered their spectra integrated over 128-s time intervals. These spectra are fitted with a simple model consisting of two components, that represent contributions of the boundary layer and the accretion disk. Also included in the model was the Gaussian line at the energy $6.4 \mathrm{keV}$. It is not energetically important but does significantly improve the quality of the fit. The line parameters will not be considered here. The shape of the boundary layer spectrum was approximated by comptt model (XSPEC fitting package) with the bestfit parameters obtained in 


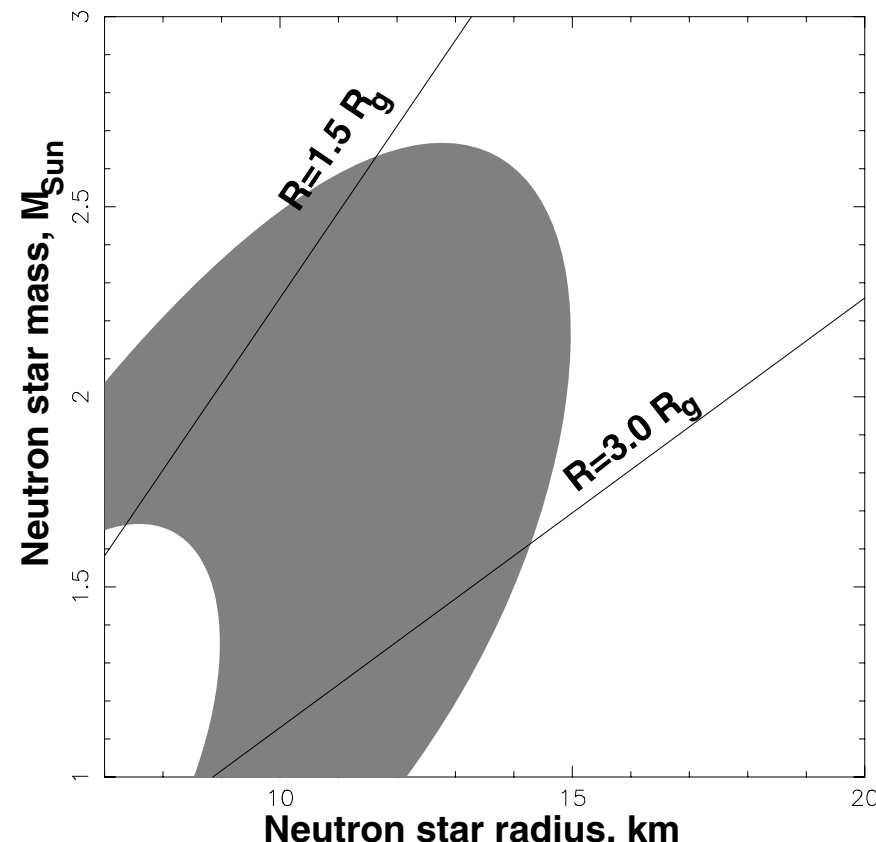

Fig. 3. Constraints on the mass and radius of the neutron star in bright LMXBs. Solid lines denote the regions where neutron star radius $R$ equals $1.5 R_{\mathrm{g}}$ (radius of photon orbit in Schwarzschild metric) and $3.0 R_{\mathrm{g}}$ (radius of last stable orbit of an accretion disk in the Schwarzschild metric).

Sect. 3. For the accretion disk spectrum we adopt the multicolor disk blackbody of Shakura \& Sunyaev (1973) and Mitsuda et al. (1984) (diskbb model in XSPEC fitting package).

The quality of the spectral fits is uniformly good on the horizontal and normal branches of the Z-track in the color-color diagram, with the reduced $\chi^{2}$ never exceeding $\sim 1.3$ for 41 degrees of freedom. Relative deviations of the model from the data does not exceed approximately one percent. This is illustrated by Fig. 4, showing two spectra of Cyg X-2 at the beginning of the horizontal branch and at the end of the normal branch.

The model is obviously oversimplified. First, it relies on the assumption about the shape of the boundary layer spectrum. This seems to have been established rather well for the horizontal branch and for the upper part of the normal branch. For the latter, it has been shown for GX $340+0$ only, while for other sources the signal-to-noise ratio is insufficient. On the lower part of the normal branch the variability level and, consequently the signalto-noise ratio of the frequency resolved spectra, are too low to conduct a similar study for any of the sources in our sample. Secondly, we chose the diskbb model to represent the spectrum of the accretion disk. Although the model involves several well known simplifying assumptions, it has been shown to approximately reproduce the shape of the spectrum of the geometrically thin, optically thick accretion disk (Merloni et al. 2000). The bestfit color temperature obtained in this model can be related with certain accuracy to the maximum disk temperature. Therefore we should be able to separate the disk and boundary layer components with this model and to estimate their respective fluxes, as well as the characteristic temperature in the inner disk. Keeping the limitations of the spectral model in mind, we will attempt to relate the motion of a source along the Z-track to the changes in the values of the physically meaningful parameters. The most important difference between our approach and similar studies undertaken previously is in a priori knowledge of the shape of the boundary layer spectrum.

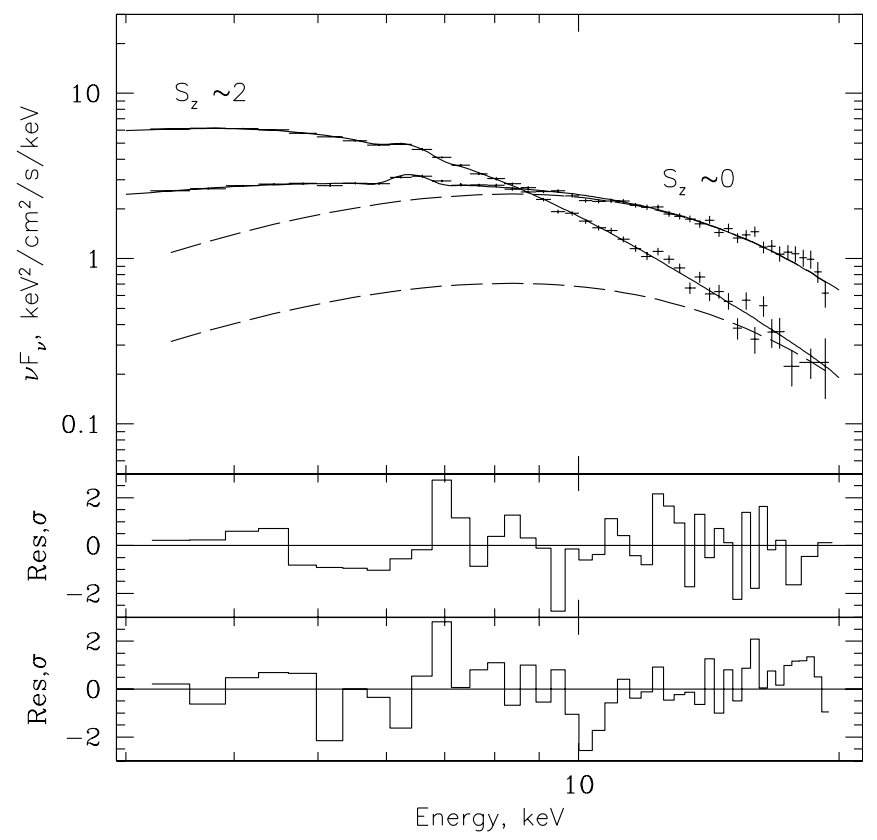

Fig. 4. Spectra of Cyg X-2 at the beginning of horizontal branch $\left(S_{\mathrm{z}} \sim\right.$ $0)$ and at the end of the normal branch $\left(S_{\mathrm{z}} \sim 2\right)$. Solid lines are best fit models of the spectra. Dashed lines denote the boundary layer components.

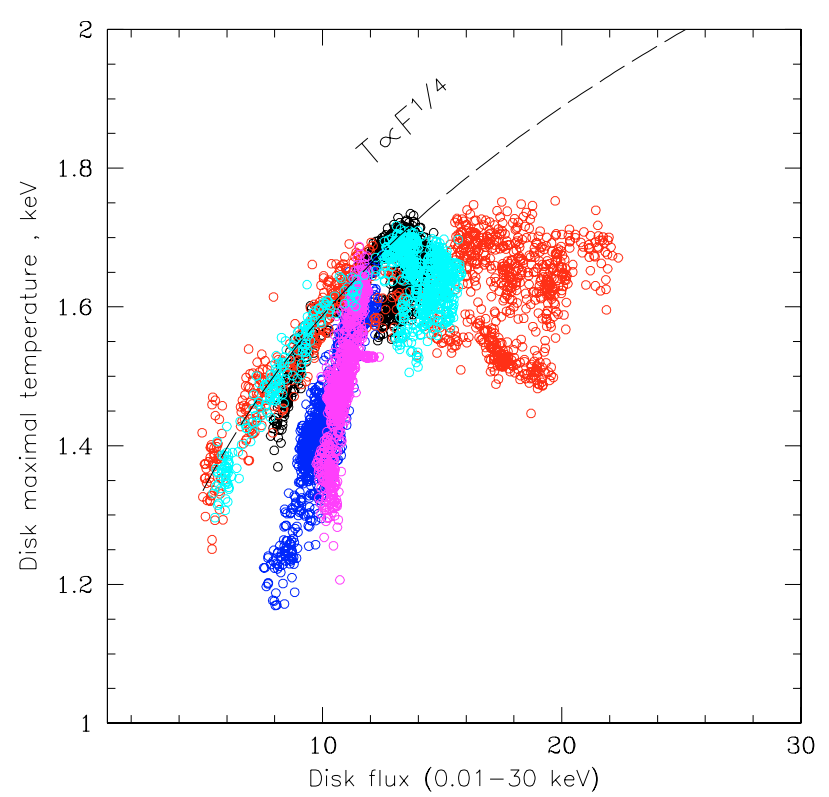

Fig. 5. Dependence of the disk temperature on its flux for five Z-sources. The units of flux are $10^{-9} \mathrm{erg} / \mathrm{s} / \mathrm{cm}^{2}$. Regions corresponding to the horizontal and normal branches are marked. The dashed curved line shows $T \propto F_{\text {disk }}^{1 / 4}$ dependence. For GX 5-1, GX 340+0, GX 17+2, and Sco X-1 the flux values were scaled down by the factor of $1.3,1.4,1.4$, and 13, respectively to match Cyg X-2 points at the end of the horizontal branch.

\subsection{Accretion disk}

The relation between the accretion disk temperature and its flux for all five Z-sources is shown in Fig. 5 (the disk flux and not the total flux is plotted along the $x$-axis). For three of them (GX 5-1, Cyg X-2, and GX 340+0) the disk temperature on the horizontal branch follows the $T \propto F_{\text {disk }}^{1 / 4}$ law approximately, which is to be expected for a standard accretion disk. For Sco X-1 and 
GX $17+2$, the temperature increases when the disk flux is rizing with the law steeper, then $T \propto F_{\text {disk }}^{1 / 4}$ law. For all sources the monotonic relation between the disk temperature and the disk flux breaks down on the normal branch, and further along the Z-track the temperature either remains constant or decreases with the disk flux.

The significant deviation from $T \propto F_{\text {disk }}^{1 / 4}$ law near the transition from the horizontal to the normal branch indicates that the simple accretion disk model in the form represented by XSPEC's diskbb becomes inapplicable. The exact reason for this is not clear. It may happen due to rather small modifications to the model, e.g. violation of the assumption that the effect of Comptonization can be represented by the single value of the spectral hardening factor, constant over the extent of the inner disk, as well as along the Z-track in the color-color diagram. Alternatively, this might be an indication of the more significant breakdown of the standard geometrically thin, optically thick picture of the accretion disk. This would point to the significant modification of the structure and geometry of the accretion flow near the transition from the horizontal to the normal branch, presumably corresponding to $\dot{M} \sim \dot{M}_{\text {Edd }}$.

Knowledge of the boundary layer and the accretion disk spectra allows one to (crudely) estimate the scale-height of the disk $H / R$ in the region of the main energy release. Indeed, for the radiation-pressure supported accretion disk, its height is determined by the equality of the radiation pressure and the gravitational force (see e.g. Shakura \& Sunyaev 1973). For a geometrically thin accretion disk, the gravitational force near the neutron star is $\sim R / H$ times smaller than on the neutron star surface. Assuming that the radiation transfer conditions are similar in the disk and in the boundary layer (i.e. the color hardening factors are similar, see Sect. 4$)$, we find $H / R \sim\left(T_{\text {disk }} / T_{\mathrm{BL}}\right)^{4} \sim$ $0.1-0.2$, which is comparable to the predictions of the simple disk theory for $\dot{M} \sim \dot{M}_{\text {Edd }}$ (Shakura \& Sunyaev 1973, 1976). On the other hand, this estimate can be considered as a crude consistency check for the obtained values of the boundary layer and disk temperatures.

\subsection{Boundary layer}

The dependencies of the BL contribution to the total X-ray emission on the position on the Z-track are plotted in Fig. 6. The coordinate along the Z-track was defined to be proportional to the hard color with the reference points $S_{Z}=0,1,2$ defined as shown in Fig. 1.

Statistical uncertainties in the values of the BL fraction are small and can be neglected, as confirmed by the dispersion of the points in Fig. 6. More important are the systematic ones associated with the imprecise knowledge of the shape of the $\mathrm{BL}$ spectrum and its possible variations along the Z-track. In order to probe the amplitude of these uncertainties on the horizontal branch, we explored the range of $1 \sigma$ errors of the parameters of the comptt model obtained in the Sect. 3 . The associated change of the boundary layer fraction did not exceed $\approx 0.05-0.07$ in the units of Fig. 6 . As for the normal branch, there is some difference between the frequency resolved spectra of GX $340+0$ on the horizontal and normal branches, the latter being better described by the Wien spectrum with $k T=2.4 \mathrm{keV}$ than by the spectrum of saturated Comptonization (Fig. 2). This difference could be related to the weak dependence of the boundary layer spectrum on the global mass accretion rate (Gilfanov et al. 2003). Substitution of the comptt model by the blackbody or Wien spectrum with $k T=2.4 \mathrm{keV}$, decreases the boundary

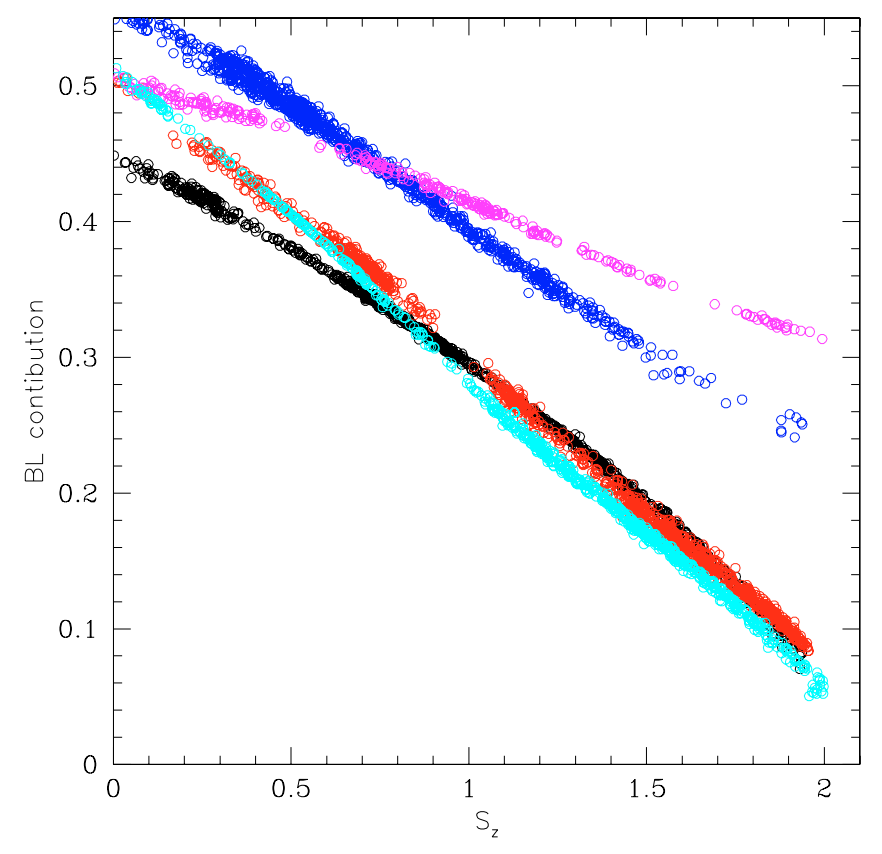

Fig. 6. Dependence of the boundary layer contribution to the total X-ray emission of $\mathrm{Z}$ sources as a faction of the position on their Z-diagrams.

layer fraction by $\approx 0.1$. As is clear from these numbers, systematic uncertainties might change the Fig. 6 in some details but does not affect the general trend in any significant way.

Figures 4 and 6 suggest that the boundary layer fraction decreases along the Z-track and is smaller on the normal branch than on the horizontal branch. As demonstrated above, this conclusion is rather robust, as long as the assumption regarding the constancy of the boundary layer spectrum is approximately correct. Even if the diskbb model is no longer applicable to the normal branch spectra, the $\sim 5$-fold decrease of the $E \gtrsim 15 \mathrm{keV}$ flux between $S_{\mathrm{z}}=0$ and $S_{\mathrm{z}}=2$ effectively constrains the boundary layer contribution on the normal branch (Fig. 6). As the variability at $f \gtrsim 1 \mathrm{~Hz}$ is primarily associated with the boundary layer emission, the decrease in the boundary layer fraction along the Z-track also explains the well-known decrease in the level of aperiodic and quasi-periodic variability.

Although no complete physical interpretation of the observed behavior can be offered, we mention several possibilities. One of these is that the general structure of the accretion flow does not change significantly and that $\sim 50 \%$ of the energy is always released on, or very close to, the neutron star surface. The apparent decrease in the boundary layer fraction on the normal branch is a result of its geometrical obscuration by, for example, the geometrically thickened accretion disk. An alternative possibility is that at high values of the mass accretion rate $\dot{M} \sim \dot{M}_{\text {Edd }}$, a significant modification of the accretion flow structure might occur, and its division into two geometrically distinct parts - boundary layer and accretion disk - might become inapplicable. Namely, due to non-negligible pressure effects, the deceleration of the orbital motion of the accreting matter from Keplerian frequency to the neutron star spin frequency would take place in a geometrically extended region with the radial extent of $\Delta R \sim R_{\mathrm{NS}}$. In this case, the observed decrease in the boundary layer fraction could reflect an actual decrease in the fraction of the energy released on the neutron star surface with the rest of the energy being released in the extended transition region. Finally, it is also possible that the apparent decrease in the boundary layer fraction, as well as the peculiar behavior of 
the accretion disk temperature (Fig. 5), is the consequence of the complete breakdown of the model on the normal branch of the color-color diagram.

Sco X-1 and GX 17+2 appear to have higher boundary layer fractions at the end of the normal branch than three other sources. Another indication of the possibly higher contribution of the BL emission at $S_{\mathrm{z}} \sim 2$ in Sco X-1 and GX 17+2 is that they typically show stronger fast variability at the normal and flaring branches than do Cyg X-2, GX 340+0 and GX 5-1 (cf. Sco X-1 -like and Cyg X-2 -like sources, Hasinger \& van der Klis 1989; Kuulkers et al. 1994; Jonker et al. 2000; Piraino et al. 2002; Homan et al. 2002). The observed difference can be caused, for example, by different inclination angles in these systems, as indicated by optical data (Fomalont et al. 2001; Steeghs \& Casares 2002; Crampton \& Cowley 1976; Orosz \& Kuulkers 1999; Kuulkers et al. 1994). It should be mentioned, though, that the appearance of Fig. 6 is a direct consequence of the definition of the $S_{\mathrm{z}}$ coordinate, in particular, the definition of the $S_{\mathrm{z}}=2$ reference point as the turning point from the normal to the flaring branch. If, for example, the BL fraction were plotted against the hard color, all five sources would almost be indistinguishable.

\subsection{Shape of the Z-diagram}

Motivated by these results, we used the two-component spectral model introduced in the beginning of this section to explain the behavior of the Z-sources on the color-color diagram. For the accretion disk spectral component we instead adopt here the general relativistic accretion disk model by Ebisawa et al. (1991) (the grad model in the XSPEC fitting package), which explicitly includes dependence on the mass accretion rate. The mass of the compact object and binary system inclination were fixed at $M_{\mathrm{NS}}=1.4 M_{\odot}$ and $i=60^{\circ}$. Using this two-component spectral model, we can calculate the position in the color-color diagram as a function of the mass accretion rate and the boundary layer fraction. This simple model is an attempt to understand general tendencies of the Z-diagram, rather than to construct its precise quantitative description. In particular, the fact is ignored, that the relation between the accretion disk temperature, and its flux is more complex than predicted by the standard model of the geometrically thin disk (Fig. 5).

The results are presented in Fig. 7. The general shape of the Z-track can be reproduced in the model as a result of the variation of two parameters - the mass accretion rate and BL fraction. The mass accretion rate increases along the Z-track. The Z-shape of the track is defined by the variation in the BL fraction, which decreases along the normal branch from the value of $\sim 50 \%$ expected in the "standard" theories to a small number of the order of $\sim$ zero at the end of the normal branch.

Quantitatively, the exact value of the $\dot{M}$, corresponding to the transition from the horizontal to the normal branches depends on the disk model ( $\mathrm{grad}$ ) parameters: the binary system inclination, the mass of the neutron star, and the spectral hardening factor. For our choice of parameters, it equals $\dot{M} \sim 2 \times 10^{18} \mathrm{~g} / \mathrm{s}$, i.e. is of the order of the Eddington critical value for a $1.4 M_{\odot}$ neutron star, when we can expect that the structure of the inner accretion flow can start to change. It is interesting to note that similar conclusions (decrease of the observable NS emission due to possible inflation of the inner disk) were obtained from studies of simultaneous observations of Z-sources in radio, optical UV, and X-ray ranges (e.g. Hasinger et al. 1990; Vrtilek et al. 1990)

The location of the horizontal branch on the color-color diagram depends on the boundary layer fraction at smaller $\dot{M}$.

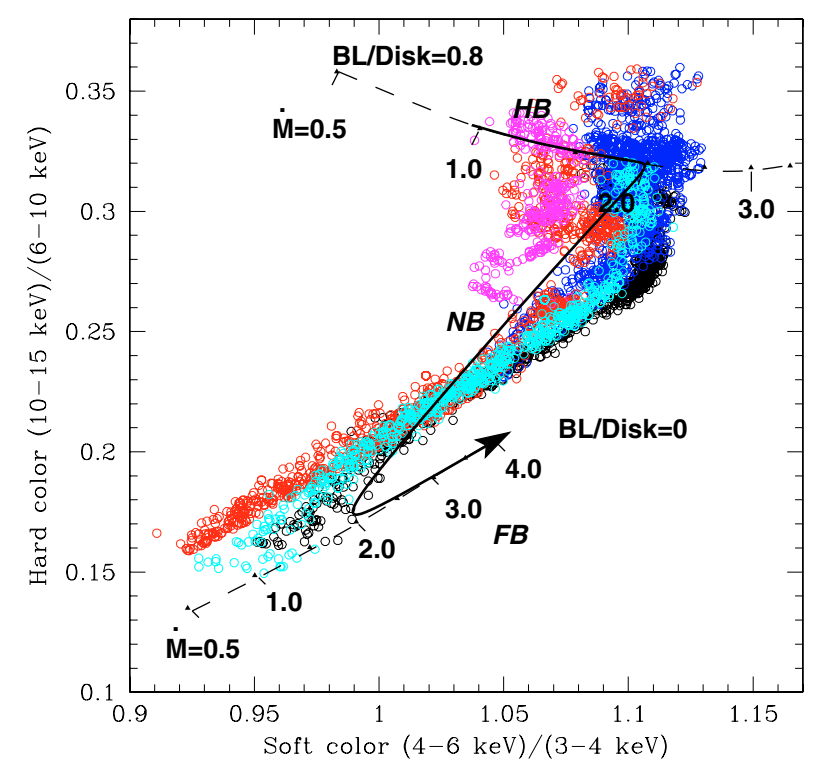

Fig. 7. The color-color diagram of 5 Z-sources. Overlayed on the data are the tracks predicted by the model (Sect. 5.3). The thick two dashed lines show evolution of the colors with change of the accretion rate in the disk for two different values of the BL fraction: $44 \%$ (upper) and zero (lower) The values of the mass accretion rates $\dot{M}$ of the disk component are marked in units of $10^{18} \mathrm{~g} / \mathrm{s}$. The thick solid line shows the $\mathrm{Z}$ track with transition mass accretion rate $2 \times 10^{18} \mathrm{~g} / \mathrm{s}$.

Theoretically, it could be used to determine the latter from observations that would be a big help for the theory. In particular, our spectral model suggests $F_{\mathrm{BL}} / F_{\mathrm{AD}} \sim 0.8$. In practice, however, the direct comparison of this number with theoretical predictions for the energy release in the disk and boundary layer is complicated, because the observed flux ratio $F_{\mathrm{BL}} / F_{\text {disk }}$ is modified by geometrical factors and by the anisotropy of the AD and BL emission diagrams.

\section{Summary}

Using the archival data of RXTE observations of several bright LMXBs, we studied the boundary layer emission and the structure of the accretion flow in these sources. Our sample included 2 atoll sources in the soft spectral state and 5 (all but one) Z-sources. Their luminosities range from $\sim 10^{37} \mathrm{erg} / \mathrm{s}$ (atoll sources) to $\sim 2 \times 10^{38} \mathrm{erg} / \mathrm{s}$ (Z-sources). Our results are summarized below.

- We constructed Fourier-frequency resolved spectra (energy spectra of variable emission) at $f \gtrsim 1-5 \mathrm{~Hz}$ for five sources, which data combines sufficient timing and spectral resolution. Within the statistical uncertainties, all the spectra have the same shape (Fig. 2). This is a remarkable result, considering that the average spectra of the atoll and Z-sources differ significantly and that their luminosities range from $\sim 10^{37} \mathrm{erg} / \mathrm{s}$ (atoll sources) to $\sim 2 \times 10^{38} \mathrm{erg} / \mathrm{s}$ (Z-sources). This result fits in the picture suggested by Gilfanov et al. (2003) and briefly outlined in the Introduction. It implies, in particular, that the frequency-resolved spectrum equals the energy spectrum of the boundary layer emission. The following is based on this assumption.

- The boundary layer spectrum in atoll sources and on the horizontal branch of Z-sources (i.e. at presumably subEddington accretion rates) can be approximated by the spectrum of saturated Comptonization (Sect. 3). Its high energy 
$(E>10 \mathrm{keV})$ cut-off corresponds to the blackbody spectrum with a temperature of $k T \sim 2.4 \mathrm{keV}$ (Fig. 2).

- Assuming that the boundary layer is supported by radiation pressure we constrained the neutron star mass and radius. For a $1.4 M_{\odot}$ neutron star and the spectral hardening factor of $1.6-1.8$, the NS radii are in the range of $R_{\mathrm{NS}} \sim 9-14 \mathrm{~km}$ (Fig. 3).

- We attempted to relate the motion of Z-sources along the Z-track to changes in the values of the physically meaningful parameters. Our results tentatively suggest that the contribution of the boundary layer component to the observed emission decreases along the Z-track from the conventional value of $\sim 50 \%$ on the horizontal branch to a rather small number at the end of the normal branch. The main difference between our approach and previous attempts is in the a priori knowledge of the shape of the boundary layer spectrum. This allowed us to avoid ambiguity in the spectral decomposition into boundary layer and disk components, which was one of the major problems in previous studies.

Acknowledgements. Authors thank Rashid Sunyaev, Nail Inogamov, Eugene Churazov, Juri Poutanen, and Valery Suleimanov for useful discussions. This research made use of data obtained through the High Energy Astrophysics Science Archive Research Center Online Service, provided by the NASA/Goddard Space Flight Center.

\section{References}

Asai, K., Dotani, T., Mitsuda, K., et al. 1994, PASJ, 46, 479

Barret, D. 2001, AdSpR, 28, 307

Bhattacharyya, S., Bhattacharya, D., \& Thampan, A. 2001, MNRAS, 325, 989

Bradshaw, C. F., Geldzahler, B. J., \& Fomalont, E. B. 2003, ApJ, 592, 486

Bradt, H., Rotshild, R., \& Swank, J. 1993, A\&AS, 97, 355

Crampton, D., \& Cowley, A. P. 1976, ApJ, 207, L171

Dieter, S., Vaughan, B., Kuulkers, E., et al. 2000, A\&A, 353, 203

Di Salvo, T., Robba, N., Iaria, R., et al. 2001, ApJ, 554, 49

Done, C., Zycki, P. T., \& Smith, D. A. 2002, MNRAS, 331, 453

Ebisawa, K., Mitsuda, K., \& Hanawa, T. 1991, ApJ, 367, 213

Fomalont, E. B., Geldzahler, B. J., \& Bradshaw, C. F. 2001, ApJ, 558, 283

Gilfanov, M., Churazov, E., \& Revnivtsev, M. 2000, MNRAS, 316, 923

Gilfanov, M., Revnivtsev, M., \& Molkov, S. 2003, A\&A, 410, 217

Gilfanov, M., \& Arefiev, V. 2005, MNRAS, submitted [arXiv: astro-ph/0501215]

Goldman, I. 1979, A\&A, 78, L15

Grebenev, S., \& Sunyaev, R. 2002, Astron. Lett., 28, 150

Haberl, F., Stella, L., White, N. E., Gottwald, M., \& Priedhorsky, W. C. 1987, ApJ, 314, 266

Hasinger, G., \& van der Klis, M. 1989, A\&A, 225, 79

Hasinger, G., van der Klis, M., Ebisawa, K., Dotani, T., \& Mitsuda, K. 1990, A\&A, 235, 131

Homan, J., van der Klis, M., Jonker, P. G., et al. 2002, ApJ, 568, 878

Iaria, R., di Salvo, T., Robba, N. R., et al. 2004, NuPhS, 132, 608
Inogamov, N., \& Sunyaev, R. 1999, Astr. Lett., 25, 269

Jonker, P., van der Klis, M., Wijnands, R., et al. 2000, ApJ, 537, 374

Kahn, S. M., Seward, F. D., \& Chlebowski, T. 1984, ApJ, 283, 286

Kluzniak, W. 1988, Ph.D. Thesis

Kuulkers, E., van der Klis, M., Oosterbroek, T., et al. 1994, A\&A, 289, 795

Kuulkers, E., Parmar, A. N., Owens, A., Oosterbroek, T., \& Lammers, U. 1997, A\&A, 323, L29

Langmeier, A., Hasinger, G., \& Truemper, J. 1990, A\&A, 228, 89

Lewin, W., Lubin, L., Tan, J., et al. 1992, MNRAS, 256, 545

Lewin, W. H. G., van Paradijs, J., \& Taam, R. E. 1993, SSRv, 62, 223

London, R. A., Howard, W. M., \& Taam, R. E. 1986, ApJ, 306, 170

Magdziarz, P., \& Zdziarski, A. A. 1995, MNRAS, 273, 837

Marshall, H. L. 1982, ApJ, 260, 815

Mendez, M., van der Klis, M., van Paradijs, J., et al. 1997, ApJ, 485, 37

Mendez, M., van der Klis, M., Ford, E., et al. 1999, ApJ, 511, L49

Mendez, M., van der Klis, M., \& Ford, E. 2001, ApJ, 561, 1016

Miller, M. C., Lamb, F., \& Psaltis, D. 1998, ApJ, 508, 791

Mitsuda, K. 1988, AdSpR, 8, 391

Mitsuda, K., \& Tanaka, Y. 1986, in The evolution of galactic X-ray binaries; Proc. of the NATO Advanced Research Workshop, Rottach-Egern (D. Reidel Publishing Co.), 195

Mitsuda, K., Inoue, H., Koyama, K., et al. 1984, PASJ, 36, 741

Orosz, J. A., \& Kuulkers, E. 1999, MNRAS, 305, 132

Penninx, W., Damen, E., van Paradijs, J., Tan, J., \& Lewin, W. H. G. 1989, A\&A, 208, 146

Piraino, S., Santangelo, A., \& Kaaret, P. 2002, ApJ, 567, 1091

Popham, R., \& Sunyaev, R. 2001, ApJ, 547, 355

Psaltis, D., Lamb, F. K., \& Miller, G. S. 1995, ApJ, 454, L137

Revnivtsev, M., Gilfanov, M., \& Churazov, E. 1999, A\&A, 347, 23

Revnivtsev, M. G., Borozdin, K. N., Priedhorsky, W. C., \& Vikhlinin, A. 2000, ApJ, 530, 955

Revnivtsev, M., Gilfanov, M., Churazov, E., \& Sunyaev, R. 2001, A\&A, 372, 138

Ross, R., \& Fabian, A. 1996, MNRAS, 281, 637

Schulz, N. S., \& Wijers, R. A. M. J. 1993, A\&A, 273,

Shakura, N. I., \& Sunyaev, R. A. 1973, A\&A, 24, 337

Shakura, N. I., \& Sunyaev, R. A. 1976, MNRAS, 175, 613

Shakura, N. I., \& Sunyaev, R. A. 1988, Ad. Sp. R., 8, 135

Shimura, T., \& Takahara, F. 1995, ApJ, 331, 780

Sibgatullin, N., \& Sunyaev, R. 2000, Astr. Lett., 26, 699

Steeghs, D., \& Casares, J. 2002, ApJ, 568, 273

Strohmayer, T. 2002, Ad. Sp. R., 28, 511

Syunyaev, R. A., \& Shakura, N. I. 1974, SvA, 18, 60

Sunyaev, R., \& Titarchuk, L. 1980, A\&A, 86, 121

Sunyaev, R., \& Shakura, N. 1986, SvAL, 12, 117

Tanaka, Y., \& Shibazaki, N. 1996, ARA\&A, 34, 607

Titarchuk, L. 1994, ApJ, 429, 340

Titarchuk, L., \& Shaposhnikov, N. 2002, ApJ, 570, L25

Toor, A., Seward, F. D., Cathey, L. R., \& Kunkel, W. E. 1970, ApJ, 160, 209

van der Klis, M. 1986, Lecture Notes in Physics, 266, 157

van der Klis, M. 2000, ARA\&A, 38, 717

van der Klis, M. 2001, ApJ, 561, 934

Vaughan, B., van der Klis, M., Lewin, W., et al. 1994, ApJ, 421, 738

Vaughan, B., van der Klis, M., Lewin, W., et al. 1999, A\&A, 343, 197

Vrtilek, S. D., Raymond, J. C., Garcia, M. R., et al. 1990, A\&A, 235, 162

White, N., Stella, L., \& Parmar, A. 1988, ApJ, 324, 363 